1 Hacettepe Journal of Mathematics and Statistics

holume 48 (2) (2019), 564-579

Research ARticle

\title{
Comparing Bhattacharyya and Kshirsagar bounds with bootstrap method
}

\author{
M. Khorashadizadeh ${ }^{* \dagger}\left(\mathbb{D}\right.$, S. Nayeban ${ }^{\ddagger}\left(\mathbb{D}\right.$, A.H. Rezaei Roknabadi ${ }^{\S}\left({ }^{\circledR}\right.$ and G.R. \\ Mohtashami Borzadaran $₫$ (iD
}

\begin{abstract}
In the class of unbiased estimators for the parameter functions, the variance of estimator is one of the basic criteria to compare and evaluate the accuracy of the estimators. In many cases the variance has complicated form and we can not compute it, so, by lower bounds, we can approximate it. Many studies have been done on the lower bounds for the variance of an unbiased estimator of the parameter. Another common and popular method that is used in many statistical problems such as variance estimation, is bootstrap method. This method has some advantages and disadvantages that must be careful when using them. In this paper, first we briefly introduce the two famous lower bounds named "Kshirsagar" (one parameter case) and "Bhattacharyya" (one and multi parameter case) bounds and then we extend the Kshirsagar bound in multi parameter case. Also, by giving some examples in different distributions, we compare one and multi parameter Bhattacharyya and Kshirsagar lower bounds with bootstrap method for approximating the variance of the unbiased estimators and show that the mentioned bounds have a better performance than bootstrap method.
\end{abstract}

Keywords: Bhattacharyya bound, Bootstrap method, Cramer-Rao bound, Hammersley-Chapman-Robbins bound, Fisher information, Kshirsagar bound.

Mathematics Subject Classification (2010): 65D20, 62N05, 62F99, 62P30.

Received: 12.03.2017 Accepted : 28.06.2018 Doi: 10.15672/HJMS.2018.618

\footnotetext{
*Department of Statistics, University of Birjand, Birjand-Iran, Email: m.khorashadizadeh@birjand.ac.ir,

$\dagger$ Corresponding Author

$\ddagger$ Department of Statistics, Ferdowsi University of Mashhad, Mashhad-Iran, Email: Samira.nayeban@yahoo.com

$\S$ Department of Statistics, Ferdowsi University of Mashhad, Mashhad-Iran, Email: rezaei@um.ac.ir

IDepartment of Statistics, Ferdowsi University of Mashhad, Mashhad-Iran, Email: grmohtashami@um.ac.ir
} 


\section{Introduction}

One of the main branches of statistical inference is estimation theory which introduce different estimators of unknown parameters and verify various properties of estimators such as unbiasedness, consistency and efficiency. In estimation theory, one of the fundamental things about accuracy of an estimator is finding a good lower bound for the variance of the estimator. In many cases the variance has complicated form and we can not compute it, and researchers are looking for ways to approximate its value. The variance of unbiased estimators of the parameter functions, is one of the basic criteria to compare and evaluate the accuracy of the estimators. In addition, the variance of unbiased estimators is one of the main components of statistical inference such as hypothesis testing and confidence intervals.

One of the most popular methods for approximating the variance of estimators which is introduced by Efron (1979) is bootstrap method. This method has some advantages and disadvantages that must be careful when using them. The other common method for estimating the variance of the estimator, is using the lower bounds. The important point of these bounds is that in many cases, they are too close to the actual value of the variance of estimator and therefore they are considered as a good approximation for the variance of the estimators. Some well-known and applicable lower bounds are CramerRao (by Rao, 1945 and Cramer, 1946), Bhattacharyya (by Bhattacharyya, 1946, 1947), Hammersley-Chapman-Robbins (by Hammersley, 1950 and Chapman and Robbins, 1951) and Kshirsagar (by Kshirsagar, 2000).

In this paper the extended version of Kshirsagar bound in multi parameter case is proposed and it is proven that the new bound is increasing with respect to its order. Also, by focusing on Bhattacharyya and Kshirsagar bounds, we compare these bounds in one and multi parameter cases, with bootstrap method for approximating the variance of unbiasd estimators via some examples. It should be noted that our comparisons show that the Bhattacharyya and Kshirsagar bounds are much more better methods with respect to bootstrap method for approximating the variance of the unbiased estimators.

\section{One parameter Bhattacharyya and Kshirsagar bounds}

In this section, we briefly introduce the structure of one parameter Bhattacharyya and Kshirsagar lower bounds.

\subsection{Bhattacharyya lower bound.}

One of the most famous lower bounds for the variance of estimators which has many applications in various fields, is Cramer-Rao bound. But this inequality states that under certain conditions, the variance of estimators can not be less than a certain value and the fact that how much the variance is greater than the quantity, is not considered. In fact the Cramer-Rao lower bound is an insufficient amount for the actual amount of the variance of estimator even for large samples. Thus we need a better Bound than Cramer-Rao bound.

Bhattacharyya $(1946,1947)$ under some regularity conditions, obtained a series of lower bounds for any unbiased estimator of parameter functions. If $X$ has a probability density function $f(X \mid \theta)$ and $T(X)$ be an unbiased estimator of $g(\theta)$ then the Bhattacharyya bounds are defined as follows,

$$
\operatorname{Var}_{\theta}(T(X)) \geq \mathbf{J}_{\theta}^{t} \mathbf{W}^{-1} \mathbf{J}_{\theta}:=B_{k}(\theta),
$$


where $t$ refers to the transpose, $\mathbf{J}_{\theta}=\left(g^{(1)}(\theta), g^{(2)}(\theta), \ldots, g^{(k)}(\theta)\right)^{t}, g^{(j)}(\theta)=\frac{\partial^{j} g(\theta)}{\partial \theta^{j}}$ for $j=1,2, \ldots, k$ and $\mathbf{W}^{-1}$ is the inverse of the Bhattacharyya matrix, where

$$
\mathbf{W}=\left(W_{r s}\right)=\left(\operatorname{Cov}_{\theta}\left\{\frac{f^{(r)}(X \mid \theta)}{f(X \mid \theta)}, \frac{f^{(s)}(X \mid \theta)}{f(X \mid \theta)}\right\}\right)
$$

such that $E_{\theta}\left(\frac{f^{(r)}(X \mid \theta)}{f(X \mid \theta)}\right)=0$ for $r, s=1,2, \ldots, k$.

In $k \times k$ Bhattacharyya matrix $(\mathbf{W}), k$ is the order of it. It is clear that $(1,1)^{t h}$ element of the Bhattacharyya matrix is the Fisher information and if we substitute $k=1$ in (2.1), then it indeed reduces to the Cramer-Rao inequality. By using the properties of the multiple correlation coefficient, it is easy to show that as the order of the Bhattacharyya matrix $(k)$ increases, the Bhattacharyya bound becomes sharper. For details and properties of Bhattacharyya bound one can see Shanbhag (1972,1979), Blight and Rao (1974), Tanaka and Akahira (2003), Tanaka (2003, 2006), Mohtashami Borzadaran (2001, 2006), Khorashadizadeh and Mohtashami Borzadaran (2007), Mohtashami Borzadaran et al. (2010).

\subsection{Kshirsagar lower bound.}

Kshirsagar (2000) extended the Hammersley-Chapman-Robbins lower bound which was introduced by Hammersley (1950) and Chapman and Robbins (1951) in the same manner of the Bhattacharyya inequality. This bound does not need the regularity assumptions and states that for any unbiased estimator $T(X)$ of $g(\theta)$,

$$
\operatorname{Var}_{\theta}(T(X)) \geq \sup _{\phi} \lambda_{\theta}^{t} \Sigma^{-1} \lambda_{\theta}:=K_{k}(\theta)
$$

where $t$ refers to the transpose, $\lambda_{\theta}=\left(g\left(\phi_{1}\right)-g(\theta), g\left(\phi_{2}\right)-g(\theta), \ldots, g\left(\phi_{k}\right)-g(\theta)\right)^{t}$ and $\Sigma^{-1}$ is the inverse of matrix with elements as follow,

$$
\Sigma_{r s}=\operatorname{Cov}_{\theta}\left(\psi_{r}, \psi_{s}\right), \quad r, s=1,2, \ldots, k,
$$

where, $\psi_{r}=\frac{f\left(X \mid \phi_{r}\right)-f(X \mid \theta)}{f(X \mid \theta)}$ and the supremum is taken over the set of all $\phi_{i} \in \Theta,(i=$ $1,2, \ldots, k)$, satisfying,

$$
S\left(\phi_{k}\right) \subset S\left(\phi_{k-1}\right) \subset \ldots \subset S\left(\phi_{1}\right) \subset S(\theta) .
$$

In case $k=1$ the lower bound (2.3) is reduced to Hammersley-Chapman-Robbins lower bound. Kshirsagar (2000) showed that his bound is sharper than the Bhattacharyya bound with corresponding order. Although, computing the Kshirsagar bound and taking the supremums are difficult, but, nowadays, using computers makes it a little easier. Tsuda and Matsumoto (2005) by improving the Kshirsagar bound, expressed its applications in quantum theory. Qin and Nayak (2008) evaluated the Kshirsagar bound for the mean square error of predictor variable and showed that these bounds are sharper than their Bhattacharyya bounds. Nayeban et al. (2013, 2014) computed and compared Kshirsagar bounds with Bhattacharyya bounds in some applicable distributions.

\section{Comparing the Bhattacharyya and Kshirsagar bounds with bootstrap method}

In statistics literature, bootstrapping is a method for assigning measures of accuracy to sample estimates which was first introduced by Efron (1979). This technique allows estimation of the sampling distribution of almost any statistic using only very simple methods. Generally, it falls in the broader class of resampling methods. Bootstrapping is the practice of estimating properties of an estimator (such as its variance) by measuring those properties when sampling from an approximating distribution. 
In the next three examples, we compute and compare the Bhattacharyya and Kshirsagar lower bounds with bootstrap method for approximating the variance of the unbiased estimators.

3.1. Example. Suppose a random variable $X$ has a negative binomial distribution with unknown parameter $p$ and known parameter $r$ and the probability mass function as follows,

$$
P(X=x)=\left(\begin{array}{c}
x-1 \\
r-1
\end{array}\right) p^{r} q^{x-r}, \quad x=r, r+1, \cdots, r \geq 1
$$

It can be easily shown that the UMVUE of parameter $p$ is,

$$
\hat{p}=\frac{r-1}{X-1}, \quad X>1
$$

but a simple expression for the exact variance of this estimator is not proposed yet. Blight and Rao (1974)and Haldan (1945) introduced the following expression which is made by elements of Bhattacharyya matrix,

$$
\operatorname{Var}(T(\underline{X}))=p^{2} \sum_{i=1}^{\infty}\left(\begin{array}{c}
r+i-1 \\
i
\end{array}\right)^{-1} q^{i}
$$

We estimate the variance of $T(X)$ by bootstrap method with 2000,10000 and 100000 replications. Also we compute the Eq. (3.1) by the third, fourth and tenth Bhattacharyya bounds and present the results in the Table 1.

Table 1. Comparing the variance estimation of the UMVUE of $p$ in negative binomial distribution by bootstrap method and Bhattacharyya bounds.

\begin{tabular}{|cc|ccc|ccc|}
\hline \hline$r$ & $p$ & $B_{3}$ & $B_{4}$ & $B_{10}$ & $\begin{array}{c}\text { Bootstrap } \\
\text { Reps. 2000 }\end{array}$ & $\begin{array}{c}\text { Bootstrap } \\
\text { Reps. 10 }\end{array}$ & $\begin{array}{c}\text { Bootstrap } \\
\text { Reps. } 10^{5}\end{array}$ \\
\hline 10 & 0.5 & 0.013778 & 0.013800 & 0.013805 & 0.13634 & 0.013836 & 0.013911 \\
5 & 0.25 & 0.012472 & 0.012754 & 0.012973 & 0.013703 & 0.013264 & 0.012753 \\
4 & 0.1 & 0.003424 & 0.003611 & 0.003876 & 0.003967 & 0.003808 & 0.004005 \\
20 & 0.9 & 0.004089 & 0.004089 & 0.004089 & 0.004179 & 0.004103 & 0.004076 \\
\hline
\end{tabular}

In Figure 1 we compute the variance of the UMVUE of $p$ for $r=5$ by the 50 th order Bhattacharyya bound, which is so sharp. Also the variance estimation by bootstrap with 10000 replications for all values of $p$ is shown in the Figure 1. 


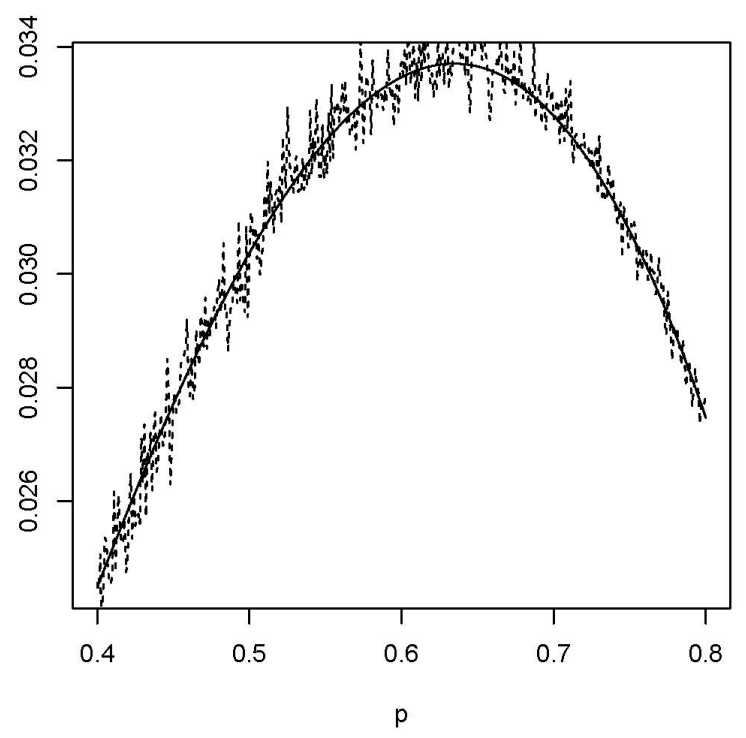

Figure 1. The variance estimation of the UMVUE of $p$ in negative binomial distribution by the 50th order Bhattacharyya bound (Solid line) and bootstrap method (Dot-Dot line). (Example 3.1)

According to the simulation studies presented in the Table 1 and Figure 1, we can see that the Bhattacharyya bounds adapt the actual amount of the variance better than the bootstrap method. It should be noted that the Bhattacharyya bounds of order more than ten, all were equal up to fifteen decimals, that indicates the precise and fast convergence of Bhattacharyya bounds.

3.2. Example. Suppose $X_{1}, \ldots, X_{n}$ be a sample of exponential distribution with mean $\theta$. One of the important factors in this distribution which is more applicable in reliability theory, is reliability function $g(\theta)=R_{\theta}(t)=e^{-\frac{t}{\theta}}$.

Pugh (1962), Basu (1964) and Patil and Wani (1966) found an unbiased estimator of this function as follows,

$$
T(\underline{X})=\left(1-\frac{t}{\sum_{i=1}^{n} X_{i}}\right)^{n-1}
$$

where $t<\sum_{i=1}^{n} X_{i}$.

Zacks and Even (1966) calculated the variance of this estimator as below,

$$
\operatorname{Var}_{\theta}(T(\underline{X}))=\frac{1}{(n-1) !} \int_{\lambda}^{\infty}\left(1-\frac{\lambda}{u}\right)^{2 n-2} e^{-u} u^{n-1} d u-e^{-2 \lambda},
$$

where $\lambda=\frac{t}{\theta}$. Computing the exact amount of this variance needs numerical methods and is not easily calculated. We present the Bhattacharyya and Kshirsagar bounds and also bootstrap approximations for the variance of the unbiased estimator of $g(\theta)$ for some values of $\lambda$ in Table 2 . 


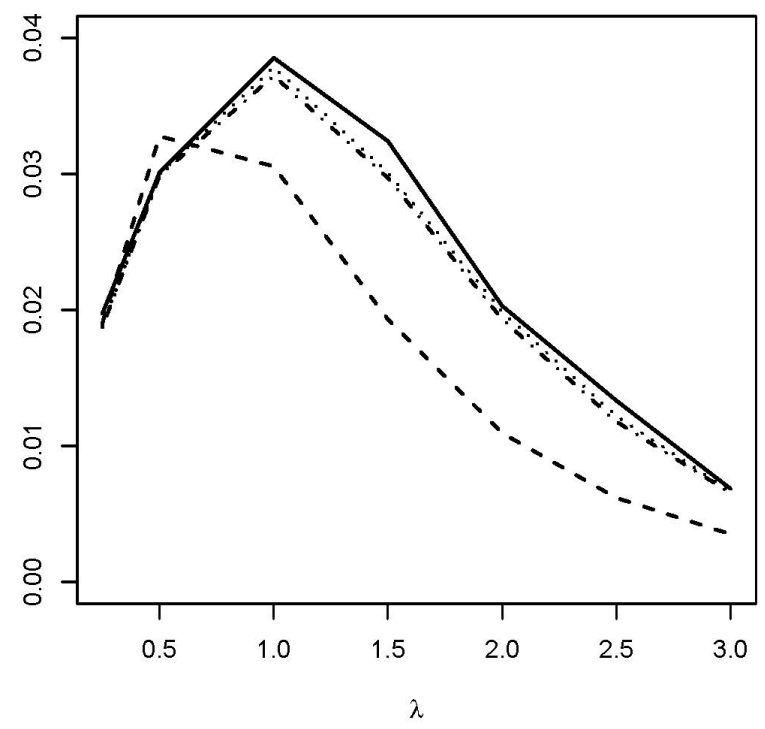

Figure 2. Comparing the exact variance (Solid line), Kshirsagar bound (Dot-Dot line), Bhattacharyya bound (Dash-Dot line) and bootstrap method (Dash-Dash line) with 50000 replications in exponential distribution for variance of unbiased estimators of the reliability function $(n=4)$. (Example 3.2)

Table 2. Approximation of the variance of any unbiased estimator of reliability function in exponential distribution with Bhattacharyya and Kshirsagar bounds and bootstrap method for $n=4$.

\begin{tabular}{|c|c|cc|cc|cc|}
\hline \hline$\lambda=\frac{t}{\theta}$ & $\operatorname{Var}_{\theta}(T(\underline{X}))$ & $B_{1}$ & $B_{2}$ & $K_{1}$ & $K_{2}$ & $\begin{array}{c}\text { Bootstrap } \\
(\text { Reps.2000) }\end{array}$ & $\begin{array}{c}\text { Bootstrap } \\
\left(\text { Reps. } 10^{4}\right)\end{array}$ \\
\hline \hline 0.25 & 0.01974 & 0.00947 & 0.01237 & 0.01582 & 0.01889 & 0.01936 & 0.01903 \\
0.5 & 0.03010 & 0.02299 & 0.02816 & 0.02828 & 0.02999 & 0.03308 & 0.03278 \\
1 & 0.03854 & 0.03383 & 0.037218 & 0.03685 & 0.03784 & 0.03039 & 0.03055 \\
1.5 & 0.03241 & 0.02800 & 0.02870 & 0.02780 & 0.03010 & 0.01901 & 0.01930 \\
2 & 0.02030 & 0.01831 & 0.01831 & 0.01901 & 0.01987 & 0.01099 & 0.01094 \\
2.5 & 0.01333 & 0.01052 & 0.01079 & 0.01091 & 0.01218 & 0.00619 & 0.00618 \\
3 & 0.00685 & 0.00577 & 0.00613 & 0.00621 & 0.00665 & 0.00358 & 0.00351 \\
\hline
\end{tabular}

Figure 2 shows the Bhattacharyya and Kshirsagar bounds and also bootstrap method for approximating the variance of the unbiased estimator of reliability function in exponential distribution. 
It is observed that the Bhattacharyya and Kshirsagar bounds offer more accurate approximations than the bootstrap method. So an important point about the Bhattacharyya and Kshirsagar bounds is approximating the variance of unbiased estimator with their help.

3.3. Example. Suppose we have a sample of size $n$ from Burr XII distribution. Since the cdf of Burr XII has closed forms, it is easy to see that its quantile $x_{q}$ of order $q$ is as follow,

$$
x_{q}=\left[(1-q)^{-\frac{1}{\theta}}-1\right]^{\frac{1}{\alpha}} .
$$

So, the median in Burr XII distribution is obtained for $q=\frac{1}{2}$ as,

$$
\text { Median }=\left[2^{\frac{1}{\theta}}-1\right]^{\frac{1}{\alpha}}
$$

Table 3. Bhattacharyya and Kshirsagar bounds for the variance of any unbiased estimator of the median in Burr XII.

\begin{tabular}{|lllllll|}
\hline \hline$\theta$ & $\alpha$ & $B_{1}$ & $B_{2}$ & $B_{3}$ & $B_{4}$ & $B_{5}$ \\
\hline 0.7 & 0.2 & 11919.92 & 170386.1 & 981303.5 & 3035420 & 6024238 \\
0.5 & 1 & 30.74899 & 45.52244 & 48.67708 & 49.05600 & 49.08512 \\
2 & 0.5 & 0.164865 & 0.261329 & 0.269681 & 0.269988 & 0.269995 \\
\hline \hline$\theta$ & $\alpha$ & $K_{1}$ & $K_{2}$ & $K_{3}$ & $K_{4}$ & $K_{5}$ \\
\hline 0.7 & 0.2 & 6619864.3 & 9130395.3 & 10992791 & 11601268.1 & 12158103 \\
0.5 & 1 & 48.24566 & 49.068000 & 49.086412 & 49.11458 & 49.11589 \\
2 & 0.5 & 0.258225 & 0.269968 & 0.2699949 & 0.2711145 & 0.2711245 \\
\hline
\end{tabular}

In Table 3, we evaluate the first five Bhattacharyya and Kshirsagar bounds for the variance of any unbiased estimator of the median in Burr XII distribution for some values of $\theta$ and $\alpha$.

Furthermore, in Figure 3 we compare the first order Bhattacharyya and first order Kshirsagar lower bounds with the bootstrap approximation of the variance of the unbiased estimator of the median in Burr XII, which indicates that, with respect to the bootstrap approximation, the Bhattacharyya and Kshirsagar lower bounds are much more nearer to the exact value of the variance. This comparison shows that the two lower bounds are good approximations for the variance of the unbiased estimators. 


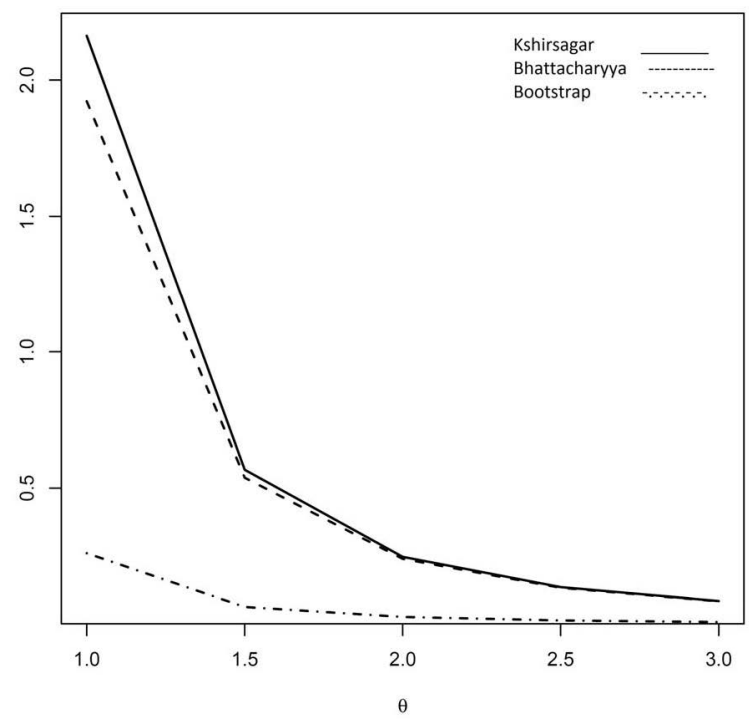

Figure 3. Comparing Bhattacharyya and Kshirsagar bounds of orders 1 and Bootstrap method (with 10000 replications) for the variance of any unbiased estimator of median in Burr XII distribution with $\alpha=1$. (Example 3.3)

\section{Multi-parameter Bhattacharyya and Kshirsagar bounds}

In this section, we first introduce the structure of multi-parameter Bhattacharyya bound and then define the multi-parameter Kshirsagar lower bound. Also, we compare the Bhattacharyya and Kshirsagar bounds with bootstrap method by two examples.

4.1. Multi-parameter Bhattacharyya lower bound. The multi-parameter version of the Bhattacharyya bound is also defined by Bhattacharyya (1947) and more considered by Pommeret (1997), Bartoszewicz (1980), Alharbi (1994) and Tanaka (2006). Bhattacharyya bounds have always been regarded as good approximations for the variance of unbiased estimators but in multi-parameter case, due to the complex structure of the matrix and the difficulty of calculating the matrix inversion, has received less attention.

One of important properties of multi-parameter Bhattacharyya bound is its convergence which has been studied by Ghosh and Sathe (1987) and Tanaka (2006). They showed that Bhattacharyya bounds converge to the variance of unbiased estimator.

Suppose $X$ has density function $f(x ; \underline{\theta})$ with an unknown parameter vector $\underline{\theta}:=$ $\left(\theta_{1}, \ldots, \theta_{r}\right) \in \Theta \subset \mathbf{R}^{r}$. Let following operators:

$$
\partial_{z}:=\left(\frac{\partial^{z}}{\partial \theta_{1}^{i_{1}}, \ldots, \partial \theta_{r}^{i_{r}}} \mid 0 \leq i_{1}, \ldots, i_{r}, \sum_{j=1}^{r} i_{j}=z\right) ; \quad z \in N
$$

and

$$
\mathbf{D}_{k}:=\left(\partial_{1}, \ldots, \partial_{k}\right)^{t},
$$

where $\partial_{z}$ is all possible partial derivatives of the form $\frac{\partial^{z}}{\partial \theta_{1}^{i_{1}}, \ldots, \partial \theta_{r}^{i_{r}}}$, which $i_{1}+\ldots+i_{r}=z$ and $i_{j}$ takes integer values $\{0,1,2, \ldots\}$ for $j=1, \ldots, r$. 
Considering the above definitions, the Bhattacharyya matrix of order $k$ is the covariance matrix of random vector,

$$
\frac{\mathbf{D}_{k} f(X ; \underline{\theta})}{f(X ; \underline{\theta})}
$$

Also note that $\partial_{z}$ has $m_{z}=\left(\begin{array}{c}z+r-1 \\ r-1\end{array}\right)$ members, so the multi-parameter Bhattacharyya matrix of order $k$, is $n_{k} \times n_{k}$ where $n_{k}=\sum_{i=1}^{k} m_{i}$.

Finally the multi-parameter Bhattacharyya inequality under suitable regularity conditions, is defined as follows:

If $T(\underline{X})$ be a real valued unbiased estimator of $g(\underline{\theta}) \in R$ then,

$$
\operatorname{Var}_{\underline{\theta}}(T(\underline{X})) \geq \eta_{\underline{\theta}} V^{-1} \eta_{\underline{\theta}}^{\prime}:=B_{r . k}(\underline{\theta})
$$

where,

1- 'denotes the transpose and $\eta_{\underline{\theta}}=\mathbf{D}_{k} g(\underline{\theta})$.

2- $V^{-1}$ is the inverse of multi-parameter Bhattacharyya matrix.

4.1. Remark. We denote the multi-parameter Bhattacharyya bound of order $k$ with $r$ unknown parameters with $B_{r . k}(\underline{\theta})$, which $B_{1 . k}$ is the one parameter Bhattacharyya bound $\left(B_{k}(\underline{\theta})\right)$.

\subsection{Multi-parameter Kshirsagar lower bound.}

In this section we present our new bound in the next theorem, which is an extension of Kshirsagar bounds.

Let $\Omega$ be a sample space and $\mathcal{A}$ be a $\sigma$-field of subsets of $\Omega$, and assume that $\Theta$ be a parameter space on open set of $\Re^{r}$ and $\boldsymbol{\theta}=\left(\theta_{1}, \ldots, \theta_{r}\right)$. Suppose that $\{f(x \mid \boldsymbol{\theta}) ; \boldsymbol{\theta} \in \Theta\}$ be a class of probability density functions or probability functions according as $x$ is continuous or discrete.

Let $\tau(\boldsymbol{\theta})$ be a real-valued function defined on $\Theta$ and $T(\boldsymbol{X})$ be an unbiased estimator of $\tau(\boldsymbol{\theta})$, i.e., $T(\boldsymbol{X})$ is a real-valued measurable function defined on $\Omega$ with property that,

$$
E_{\boldsymbol{\theta}}(T(\boldsymbol{X}))=\tau(\boldsymbol{\theta}), \quad \forall \boldsymbol{\theta} \in \Theta .
$$

For presenting lower bound for the variance of $T(\boldsymbol{X})$, first we should define some notation and symbols:

Let $\boldsymbol{\Phi}_{i}(\boldsymbol{\theta})$ be a set of subsets of parameter spaces which are defined on $\Theta$ such that for $i=1,2, \ldots$,

$$
\boldsymbol{\Phi}_{i}(\boldsymbol{\theta}):=\left(\Phi_{i_{1}}\left(\theta_{1}\right), \ldots, \Phi_{i_{r}}\left(\theta_{r}\right) \quad \mid \quad \sum_{j=1}^{r} i_{j}=i, \quad i_{j} \in\{0,1, \ldots\}\right)
$$

where $\Phi_{i_{j}}\left(\theta_{j}\right)$ is a function of $\theta_{j}$ such that $\Phi_{0}\left(\theta_{j}\right)=\theta_{j}$ for all $j=1, \ldots, r$ and

$$
S\left(\boldsymbol{\Phi}_{k}(\boldsymbol{\theta})\right) \subset \ldots \subset S\left(\boldsymbol{\Phi}_{1}(\boldsymbol{\theta})\right) \subset S(\boldsymbol{\theta}),
$$

where $S(\boldsymbol{\theta})=\{x \mid f(x \mid \boldsymbol{\theta})>0\}$. It is known that, for any $i$, there are $m_{i}=\left(\begin{array}{c}i+r-1 \\ r-1\end{array}\right)$ sets of non-negative $\left(i_{1}, \ldots, i_{r}\right)$ which satisfies $\sum_{j=1}^{r} i_{j}=i$.

For an example of such $\boldsymbol{\Phi}_{i}(\boldsymbol{\theta})$, we can take for $j=1, \ldots, r, \Phi_{i j}\left(\theta_{j}\right)=\theta_{j}+i_{j} \delta_{j}$ for some proper values of $\delta_{j}$ satisfying (4.3). For instance,

$$
\boldsymbol{\Phi}_{1}(\boldsymbol{\theta})=\left\{\left(\theta_{1}+\delta_{1}, \theta_{2}, \ldots, \theta_{r}\right),\left(\theta_{1}, \theta_{2}+\delta_{2}, \ldots, \theta_{r}\right), \ldots,\left(\theta_{1}, \theta_{2}, \ldots, \theta_{r}+\delta_{r}\right)\right\},
$$

or

$$
\begin{aligned}
\boldsymbol{\Phi}_{2}(\boldsymbol{\theta})= & \left\{\left(\theta_{1}+2 \delta_{1}, \theta_{2}, \ldots, \theta_{r}\right),\left(\theta_{1}, \theta_{2}+2 \delta_{2}, \ldots, \theta_{r}\right), \ldots,\left(\theta_{1}, \theta_{2}, \ldots, \theta_{r}+2 \delta_{r}\right),\right. \\
& \left.\left(\theta_{1}+\delta_{1}, \theta_{2}+\delta_{2}, \theta_{3}, \ldots, \theta_{r}\right), \ldots,\left(\theta_{1}, \theta_{2}, \ldots, \theta_{r-1}+\delta_{r-1}, \theta_{r}+\delta_{r}\right)\right\} .
\end{aligned}
$$


Consider the row vector $\boldsymbol{\lambda}_{k}$ as follow,

$$
\boldsymbol{\lambda}_{k}=\left(\boldsymbol{h}_{1}, \ldots, \boldsymbol{h}_{k}\right)
$$

where $\boldsymbol{h}_{i}=\left(\tau\left(\boldsymbol{\Phi}_{i}(\boldsymbol{\theta})\right)-\tau(\boldsymbol{\theta}) \mathbf{1}\right)$ is a row vector of size $m_{i}$ and $\mathbf{1}$ is a row vector of ones and therefore $\boldsymbol{\lambda}_{k}$ is a $1 \times n_{k}$ row vector where $n_{k}=\sum_{i=1}^{k} m_{i}=\sum_{i=1}^{k}\left(\begin{array}{c}i+r-1 \\ r-1\end{array}\right)$.

Let $\boldsymbol{\Psi}_{i}$, for $i=1, \ldots, k$ be a vector of form below,

$$
\boldsymbol{\Psi}_{i}=\frac{f\left(X \mid \boldsymbol{\Phi}_{i}(\boldsymbol{\theta})\right)-f(X \mid \boldsymbol{\theta})}{f(X \mid \boldsymbol{\theta})}, \quad i=1, \ldots, k,
$$

and let further, $\boldsymbol{\Sigma}_{k}$ be the covariance matrix of random vector:

$$
\left(\boldsymbol{\Psi}_{1}, \ldots, \boldsymbol{\Psi}_{k}\right) \text {. }
$$

By defining the following operator,

$$
\boldsymbol{D}_{k} f(x \mid \boldsymbol{\theta}):=\left(f\left(x \mid \boldsymbol{\Phi}_{1}(\boldsymbol{\theta})\right), \ldots, f\left(x \mid \mathbf{\Phi}_{k}(\boldsymbol{\theta})\right)\right),
$$

$\boldsymbol{\Sigma}_{k}$ is a $n_{k} \times n_{k}$ covariance matrix of random row vector

$$
\frac{\boldsymbol{D}_{k} f(X \mid \boldsymbol{\theta})}{f(X \mid \boldsymbol{\theta})}-\mathbf{1}
$$

where $\mathbf{1}$ is a row vector of ones of convenient size.

Now we present our new bound in the next theorem, which is an extension of Barankin and Kshirsagar bounds.

4.2. Theorem. If $T(\boldsymbol{X})$ be any unbiased estimator of the parameter function $\tau(\boldsymbol{\theta})$ then,

$$
\operatorname{Var}_{\boldsymbol{\theta}}(T(\boldsymbol{X})) \geq \sup \boldsymbol{\lambda}_{k} \boldsymbol{\Sigma}_{k}^{-1} \boldsymbol{\lambda}_{k}^{\prime}:=K_{r . k}(\boldsymbol{\theta}) \text { say, }
$$

where, the supremum is with respect to all $\boldsymbol{\Phi}_{i}(\boldsymbol{\theta})$ satisfying (4.3).

Proof:

It is easy to see that for any $i, E_{\boldsymbol{\theta}}\left(\boldsymbol{\Psi}_{i}\right)=0$ and therefore for any $k$,

$E_{\boldsymbol{\theta}}\left(\frac{\boldsymbol{D}_{k} f(X \mid \boldsymbol{\theta})}{f(X \mid \boldsymbol{\theta})}-\mathbf{1}\right)=\mathbf{0}$, (where $\mathbf{0}$ is a row vector of zeros of size $n_{k}$ ). We can partition the matrix of $\boldsymbol{\Sigma}_{k}$ as follow:

$$
\boldsymbol{\Sigma}_{k}=\left(\begin{array}{cccc}
\Sigma_{11} & \Sigma_{12} & \ldots & \Sigma_{1 k} \\
\vdots & \vdots & \ddots & \vdots \\
\Sigma_{k 1} & \Sigma_{k 2} & \ldots & \Sigma_{k k}
\end{array}\right)
$$

where the $m_{l} \times m_{s}$ block matrix $\Sigma_{l s}$ is of the form,

$$
\Sigma_{l s}=E_{\boldsymbol{\theta}}\left(\boldsymbol{\Psi}_{l} . \boldsymbol{\Psi}_{s}\right), \quad l, s=1, \ldots, k .
$$

Hence, the covariance between $T(\boldsymbol{X})$ and every elements of the set $\boldsymbol{\Psi}_{i}$ is as follow,

$$
\begin{aligned}
\operatorname{Cov}_{\boldsymbol{\theta}}\left(T(\boldsymbol{X}), \mathbf{\Psi}_{i}\right) & =E_{\boldsymbol{\theta}}\left(T(\boldsymbol{X}) \cdot \mathbf{\Psi}_{i}\right) \\
& =\int T(\boldsymbol{x}) \cdot f\left(x \mid \boldsymbol{\Phi}_{i}(\boldsymbol{\theta})\right) d \mu(x)-\int T(\boldsymbol{x}) \cdot f(x \mid \boldsymbol{\theta}) d \mu(x) \\
& =\tau\left(\boldsymbol{\Phi}_{i}(\boldsymbol{\theta})\right)-\tau(\boldsymbol{\theta}) \mathbf{1} \\
& =\boldsymbol{h}_{i} .
\end{aligned}
$$

Finally, it is easy to see that the multiple correlation coefficient $\left(\boldsymbol{\rho}_{k}\right)$ between $T(\boldsymbol{X})$ and $\left(\boldsymbol{\Psi}_{1}, \ldots, \boldsymbol{\Psi}_{k}\right)$ is given by,

$$
\boldsymbol{\rho}_{k}^{2}=\frac{\boldsymbol{\lambda}_{k} \boldsymbol{\Sigma}_{k}^{-1} \boldsymbol{\lambda}_{k}^{\prime}}{\operatorname{Var}_{\boldsymbol{\theta}}(T(\boldsymbol{X}))}
$$

which yields the required result. $\triangle$ 
4.3. Remark. It is well known that $K_{r .1}(\boldsymbol{\theta})$ is identical to the Barankin bound and $K_{1 . k}(\boldsymbol{\theta})$ is identical to the Kshirsagar bound $\left(K_{k}(\boldsymbol{\theta})\right)$ and finally $K_{1.1}(\boldsymbol{\theta})$ leads to the Hammersley-Chapman-Robbins lower bound.

In the next theorem we try to show that as the order of the matrix $\boldsymbol{\Sigma}_{k}$ increases the bound get sharper and sharper.

4.4. Theorem. The lower bound for the variance of $T(\boldsymbol{X})$ in Theorem 4.2, is increasing with respect to $k$, i.e.

$$
K_{r . k}(\boldsymbol{\theta}) \leq K_{r . k+1}(\boldsymbol{\theta})
$$

Proof:

Using the partition (4.5) it is straightforward that,

$$
\boldsymbol{\Sigma}_{k+1}=\left(\begin{array}{ccc}
\boldsymbol{\Sigma}_{k} & \vdots & A \\
\cdots & \cdots & \cdots \\
A^{\prime} & \vdots & B
\end{array}\right)
$$

where $A=\left(\begin{array}{c}\Sigma_{1(k+1)} \\ \vdots \\ \Sigma_{k(k+1)}\end{array}\right)$ is a $n_{k} \times m_{k+1}$ matrix and $B=\Sigma_{(k+1)(k+1)}$ is a $m_{k+1} \times m_{k+1}$ matrix.

Then, the block inverse of the matrix $\boldsymbol{\Sigma}_{k+1}$ is,

$$
\boldsymbol{\Sigma}_{k+1}^{-1}=\left(\begin{array}{ll}
C_{11} & C_{12} \\
C_{21} & C_{22}
\end{array}\right)
$$

where

$$
\begin{aligned}
& C_{11}=\left(\boldsymbol{\Sigma}_{k}-A B^{-1} A^{\prime}\right)^{-1}, \\
& C_{12}=-\boldsymbol{\Sigma}_{k}^{-1} A\left(B-A^{\prime} \boldsymbol{\Sigma}_{k}^{-1} A\right)^{-1}, \\
& C_{21}=-B^{-1} A^{\prime}\left(\boldsymbol{\Sigma}_{k}-A B^{-1} A^{\prime}\right)^{-1}, \\
& C_{22}=\left(B-A^{\prime} \boldsymbol{\Sigma}_{k}^{-1} A\right)^{-1} .
\end{aligned}
$$

Therefor the lower bound of order $k+1$ is expressed as,

$$
K_{r . k+1}(\boldsymbol{\theta})=\sup \left[\begin{array}{ll}
\boldsymbol{\lambda}_{k} & \boldsymbol{h}_{k+1}
\end{array}\right] \boldsymbol{\Sigma}_{k+1}^{-1}\left[\begin{array}{c}
\boldsymbol{\lambda}_{k}^{\prime} \\
\boldsymbol{h}_{k+1}^{\prime}
\end{array}\right],
$$

where $\boldsymbol{h}_{k+1}=\left(\tau\left(\boldsymbol{\Phi}_{k+1}(\boldsymbol{\theta})\right)-\tau(\boldsymbol{\theta}) \mathbf{1}\right)$ is a row vector of size $m_{k+1}$. Now substituting (4.9) in to (4.10) leads to,

$$
K_{r . k+1}(\boldsymbol{\theta})=\sup \left[\boldsymbol{\lambda}_{k} C_{11} \boldsymbol{\lambda}_{k}^{\prime}+\boldsymbol{h}_{k+1} C_{21} \boldsymbol{\lambda}_{k}^{\prime}+\boldsymbol{\lambda}_{k} C_{12} \boldsymbol{h}_{k+1}^{\prime}+\boldsymbol{h}_{k+1} C_{22} \boldsymbol{h}_{k+1}^{\prime}\right] .
$$

Using the lemma presented by Miller (1981) we can write $C_{11}$ as follow,

$$
C_{11}=\boldsymbol{\Sigma}_{k}^{-1}+\left(\boldsymbol{I}-\boldsymbol{\Sigma}_{k}^{-1} A B^{-1} A^{\prime}\right)^{-1} \boldsymbol{\Sigma}_{k}^{-1} A B^{-1} A^{\prime} \boldsymbol{\Sigma}_{k}^{-1},
$$

hence by substitution we have,

$$
\begin{aligned}
K_{r . k+1}(\boldsymbol{\theta})=K_{r . k}(\boldsymbol{\theta})+\sup & {\left[\boldsymbol{\lambda}_{k}\left(\boldsymbol{I}-\boldsymbol{\Sigma}_{k}^{-1} A B^{-1} A^{\prime}\right)^{-1} \boldsymbol{\Sigma}_{k}^{-1} A B^{-1} A^{\prime} \boldsymbol{\Sigma}_{k}^{-1} \boldsymbol{\lambda}_{k}^{\prime}\right.} \\
+ & \left.\boldsymbol{h}_{k+1} C_{21} \boldsymbol{\lambda}_{k}^{\prime}+\boldsymbol{\lambda}_{k} C_{12} \boldsymbol{h}_{k+1}^{\prime}+\boldsymbol{h}_{k+1} C_{22} \boldsymbol{h}_{k+1}^{\prime}\right]
\end{aligned}
$$

where the second term in right hand side is positive and the inequality is satisfied. $\triangle$

4.5. Example. Suppose $X_{1}, \ldots, X_{n}$ and $Y_{1}, \ldots, Y_{m}$ be random samples of exponential distributions with means $\theta_{1}$ and $\theta_{2}$. Tong $(1974,1975)$ found the UMVUE of $P(X<$ $Y)=\frac{\theta_{2}}{\theta_{1}+\theta_{2}}$ for the first time which is as follow, 


$$
\widehat{R}= \begin{cases}Q_{1}\left(n, m, \sum_{i=1}^{n} X_{i}, \sum_{j=1}^{m} Y_{j}\right) ; & \sum_{j=1}^{m} Y_{j} \leq \sum_{i=1}^{n} X_{i}, \\ Q_{2}\left(n, m, \sum_{i=1}^{n} X_{i}, \sum_{j=1}^{m} Y_{j}\right) ; & \sum_{j=1}^{m} Y_{j}>\sum_{i=1}^{n} X_{i},\end{cases}
$$

where,

$$
\begin{aligned}
Q_{1}(a, b, u, v) & =\sum_{i=0}^{a-2}(-1)^{i} \frac{\Gamma(a) \Gamma(b)}{\Gamma(a-i-1) \Gamma(b+i+1)}\left(\frac{v}{u}\right)^{i+1} \\
Q_{2}(a, b, u, v) & =\sum_{i=0}^{b-1}(-1)^{i} \frac{\Gamma(a) \Gamma(b)}{\Gamma(a+i) \Gamma(b-i)}\left(\frac{u}{v}\right)^{i}
\end{aligned}
$$

Computation of the variance of this estimator has been considered by many researchers. Kotz et al. (2003) computed the following expression for the UMVUE of $\widehat{\operatorname{Var}}(\widehat{R})$,

$$
\widehat{\operatorname{Var}}(\widehat{R})=(\widehat{R})^{2}-\frac{(n-1)(n-2)(m-1)(m-2)}{n^{2} m^{2} \bar{X}^{n-1} \bar{Y}^{m-1}} H(n, m, \bar{X}, \bar{Y})
$$

where, $H(n, m, \bar{X}, \bar{Y})$ is obtained by,

$$
H(n, m, \bar{X}, \bar{Y})=\iiint \int_{B}\left(\bar{X}-\frac{x_{1}+x_{2}}{n}\right)^{n-3}\left(\bar{Y}-\frac{y_{1}+y_{2}}{m}\right)^{m-3} d x_{1} d x_{2} d y_{1} d y_{2},
$$

and space $B$ is expressed as,

$$
\begin{aligned}
B= & \left\{\left(x_{1}, x_{2}, y_{1}, y_{2}\right): x_{1}+x_{2}<n \bar{X}, y_{1}+y_{2}<m \bar{Y},\right. \\
& \left.0<x_{1}<y_{1}, 0<x_{2}<y_{2}\right\} .
\end{aligned}
$$

Computing above integrals, is very difficult and should be computed by numerical methods.

In Table 4 we approximate the variance of UMVUE of $P(X<Y)$ with Bhattacharyya bounds and bootstrap method.

Table 4. Approximating the variance of UMVUE of $P(X<Y)$ with Bhattacharyya bounds (with orders $k=3,4$ ) and bootstrap method

\begin{tabular}{|c|c|c|cc|cc|}
\hline \hline$n$ & $m$ & $\rho=\frac{\theta_{1}}{\theta_{2}}$ & $k=3$ & $k=4$ & $\begin{array}{c}\text { Bootstrap } \\
\text { (2000 Replications) }\end{array}$ & $\begin{array}{c}\text { Bootstrap } \\
\text { (10000 Replications) }\end{array}$ \\
\hline \hline 5 & 5 & 0.25 & 0.01213 & 0.01224 & 0.009615 & 0.00954 \\
5 & 10 & 0.75 & 0.01924 & 0.01926 & 0.020078 & 0.019865 \\
10 & 10 & 1 & 0.01312 & 0.01312 & 0.013587 & 0.013492 \\
10 & 10 & 0.5 & 0.01049 & 0.01050 & 0.007453 & 0.007357 \\
5 & 10 & 0.25 & 0.00830 & 0.00832 & 0.008243 & 0.008291 \\
\hline
\end{tabular}

It can be seen in Figure 4 that although the Cramer-Rao bound is weaker than Bhattacharyya bounds, but it still acts better than bootstrap approximations. 


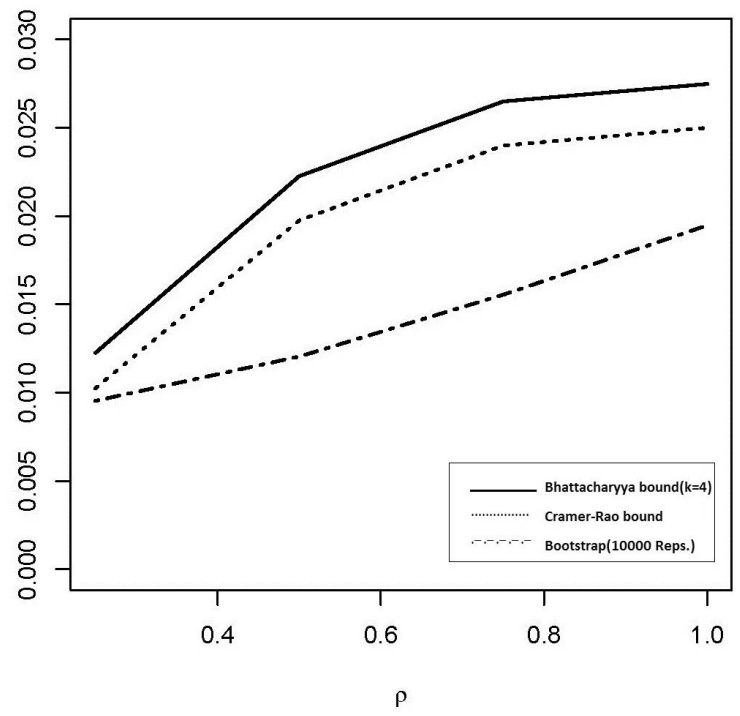

Figure 4. Approximating the variance of UMVUE of $P(X<Y)$ with Cramer-Rao and Bhattacharyya bounds and Bootstrap method in exponential distribution $(n=m=5)$.

4.6. Example. Suppose $X_{1}, \ldots, X_{n}$ be a sample of Pareto distribution with unknown parameters $\alpha$ and $\beta$. Asrabadi (1990) showed that the UMVUE of mean function in Pareto distribution is,

$$
T(\underline{X})=\frac{(n-1) ! \widehat{\beta}}{(\ln t-n \ln \widehat{\beta})^{1-n}}\left(\frac{t}{\widehat{\beta}^{n}}-1-\sum_{i=1}^{n-2} \frac{(\ln t-n \ln \widehat{\beta})^{i}}{i !}\right),
$$

where, $\widehat{\beta}=\min \left\{X_{i}\right\}$ and $t=\prod_{i=1}^{n} X_{i}$.

Unfortunately, calculating the variance of this estimator has not been discussed yet and here we try to give the best approximation of it by comparing the Kshirsagar bounds with bootstrap method. In Table 5 we present the variance approximation for the UMVUE of the mean function in Pareto distribution by Kshirsagar bounds and bootstrap method.

Table 5. Multi-parameter Kshirsagar bounds (with orders $k=1,2$ ) and bootstrap approximation for the variance of unbiased estimator of mean function in Pareto distribution with unknown $\alpha$ and $\beta$

\begin{tabular}{|c|c|c|cc|cc|}
\hline \hline$n$ & $\alpha$ & $\beta$ & $K_{2 \cdot 1}$ & $K_{2 \cdot 2}$ & $\begin{array}{c}\text { Bootstrap } \\
\text { 2000 Replications }\end{array}$ & $\begin{array}{c}\text { Bootstrap } \\
\text { 10000 Replications }\end{array}$ \\
\hline \hline 2 & 3 & 1 & 3.58847 & 4.52821 & 1.512415 & 3.79487 \\
5 & 10 & 2 & 4.00102 & 10.21191 & 7.85186 & 8.36154 \\
5 & 20 & 3 & 0.00009 & 0.00089 & 0.00012 & 0.00054 \\
10 & 25 & 5 & 0.00301 & 0.02891 & 0.00652 & 0.00541 \\
\hline
\end{tabular}


Figure 5 shows the importance of Kshirsagar bounds against the bootstrap method in approximating the variance of unbiased estimator of the mean in Pareto distribution. It can be seen that the bootstrap approximations are usually less than the actual amount of the variance, because they are less than the second order Kshirsagar bound.

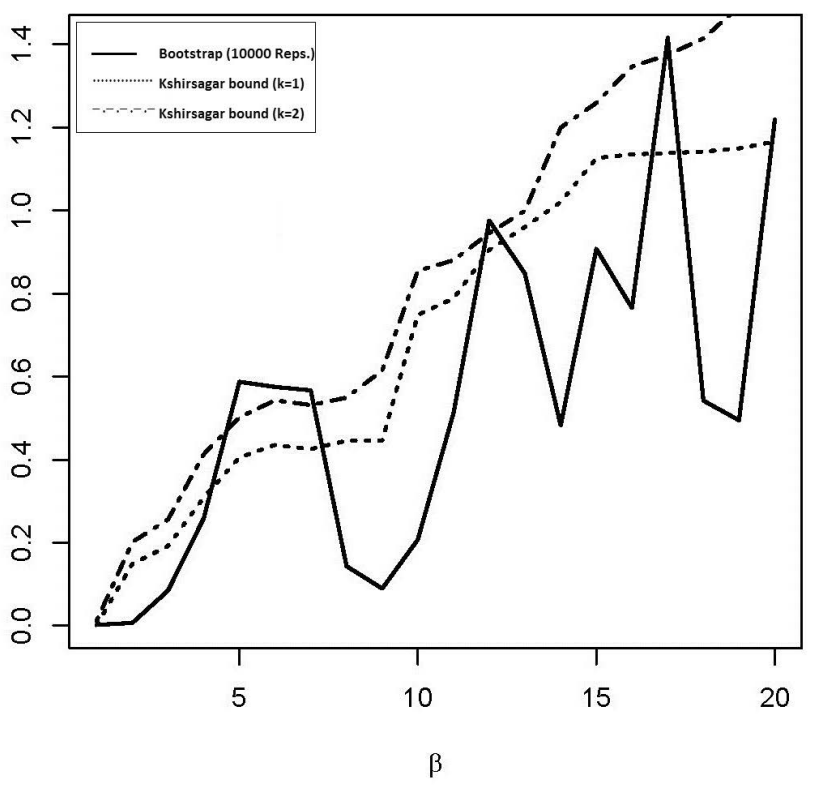

Figure 5. Approximating the variance of UMVUE of $P(X<Y)$ with Cramer-Rao and Bhattacharyya bounds and Bootstrap method in exponential distribution $(n=m=5)$.

\section{Conclusion}

In this paper, we define the Bhattacharyya and Kshirsagar bounds in both one and multi parameter cases and compute these bounds for the variance of any unbiased estimator of the parameter function in some applicable distributions. Also, we compare the Bhattacharyya and Kshirsagar lower bounds with bootstrap method in some examples. We see that the Bhattacharyya and Kshirsagar bounds are closer to the exact amount of the variance of unbiased estimators than bootstrap method.

\section{Acknowledgements}

The authors are grateful to the editors and reviewers of the manuscript.

\section{References}

[1] Alharbi, A. A. G. On the convergence of the Bhattacharyya bounds in the multiparametric case, Applicationes Mathematicae, 22, (3), 339-349, 1994.

[2] Asrabadi, B. R. Estimation in the Pareto distribution, Metrika, 37, 199-205, 1990.

[3] Bartoszewicz, J. On the convergence of Bhattacharyya bounds in the multiparameter case, Zastos. Mat., 16, 601-608, 1980. 
[4] Basu, A. P. Estimates of reliability for some distributions useful in life testing, Technometrics, 6, 215-219, 1964.

[5] Bhattacharyya, A. On some analogues of the amount of information and their use in statistical estimation, Sankhya A, 8, 1-14, 1946.

[6] Bhattacharyya, A. On some analogues of the amount of information and their use in statistical estimation II, Sankhya A, 8, 201-218, 1947.

[7] Blight, B. J. N. and Rao, R. V. The convergence of Bhattacharyya bounds, Biometrika, 61, (1), 137-142, 1974.

[8] Chapman, D. G. and Robbins, H. Minimum variance estimation without regularity assumptions. Ann. Math. Statist., 22, 581-586, 1951.

[9] Cramer, H. Mathematical Methods of Statistics, University Press, Princeton, 1946.

[10] Efron, B. Bootstrap methods: Another look at the jackknife, The Annals of Statistics, 7, 1. 1-26, 1979.

[11] Ghosh, J. K. and Sathe, S. Convergence of Bhattacharyya bounds-Revisited, Sankhya, The Indian Journal of Statistics, 49, Series A, 37-42, 1987.

[12] Haldan, J. B. S. On the method of estimating frequencies, Ann. Eugen., 11, 182-187, 1945.

[13] Hammersley, J. M. On estimating restricted parameters, J. Roy. Statist. Soc. Ser. B, 12, 192-240, 1950.

[14] Khorashadizadeh, M. and Mohtashami Borzadaran, G. R. The structure of Bhattacharyya matrix in natural exponential family and its role in approximating the variance of a statistics, J. Statistical Research of Iran (JSRI), 4, (1), 29-46, 2007.

[15] Kotz, S., Lumelski, I. P., and Pensky, M. The stress-strength Model and its Generalizations: Theory and Applications, World Scientific, 2003.

[16] Kshirsagar, A. M. An extension of the Chapman-Robbins inequality, J. Indian Statist. Assoc., 38, 355-362, 2000.

[17] Miller, K. S. On the inverse of the sum of matrices, Mathematics Magazine, 54, 2, 67-72, 1981.

[18] Mohtashami Borzadaran, G. R. Results related to the Bhattacharyya matrices, Sankhya A, 63, 1, 113-117, 2001

[19] Mohtashami Borzadaran, G. R. A note via diagonality of the 2×2 Bhattacharyya matrices, J. Math. Sci. Inf., 1, (2), 73-78, 2006.

[20] Mohtashami Borzadaran, G. R., Rezaei Roknabadi, A. H. and Khorashadizadeh, M. A view on Bhattacharyya bounds for inverse Gaussian distributions, Metrika, 72, 2, 151-161, 2010.

[21] Nayeban, S., Rezaei Roknabadi, A. H. and Mohtashami Borzadaran, G. R. Bhattacharyya and Kshirsagar bounds in generalized gamma distribution, Communications in StatisticsSimulation and Computation, 42, 5, 969-980, 2013.

[22] Nayeban, S., Rezaei Roknabadi, A.H. and Mohtashami Borzadaran, G.R. Bhattacharrya and Kshirsagar Lower Bounds for the Natural Exponential Family (NEF), Economic Quality Control, 29, 1, 63-75, 2014.

[23] Patil, G. P. and Wani, J. K. Minimum variance unbiased estimation of the distribution function admitting a sufficient statistic, Ann. Inst. Statist. Math. Tokyo 18, 39-47, 1966.

[24] Pommeret, D. Multidimensional Bhattacharyya matrices and exponential families, Journal of multivariate analysis, 63, 105-118, 1977.

[25] Pugh, E. L. The best estimate of reliability in the exponential case, Oper. Res. 11, 57-61, 1962.

[26] Qin, M. and Nayak, T. K. Kshirsagar-type lower bounds for mean squared error of prediction, Communications in Statistics - Theory and Methods, 37, 6, 861-872, 2008.

[27] Rao,C.R. Information and the accuracy attainable in the estimation of statistical parameters, Bull.Calcutta Math.Soc., 37,81-91, 1945.

[28] Shanbhag, D. N. Some characterizations based on the Bhattacharyya matrix, J. Appl. Probab., 9, 580-587, 1972.

[29] Shanbhag, D. N. Diagonality of the Bhattacharyya matrix as a characterization, Theory Probab. Appl., 24, 430-433, 1979.

[30] Tanaka, H. On a relation between a family of distributions attaining the Bhattacharyya bound and that of linear combinations of the distributions from an exponential family, Comm. Stat. Theory. Methods, 32, (10), 1885-1896, 2003. 
[31] Tanaka, H. Location and scale parameter family of distributions attaining the Bhattacharyya bound, Communications in Statistics - Theory and Methods, 35, 9, 1611-1628, 2006.

[32] Tanaka, H. and Akahira, M. On a family of distributions attaining the Bhattacharyya bound, Ann. Inst. Stat. Math.,55, 309-317, 2003.

[33] Tong, H. A note on the estimation of $\operatorname{Pr}(Y<X)$ in the exponential case, Technometrics, 16,4, 625-625, 1974

[34] Tong, H. Errata: A note on the estimation of $\operatorname{Pr}(Y<X)$ in the exponential case, Technometrics, 17, 395-395, 1975.

[35] Tsuda, Y., and Matsumoto, K. Quantum estimation for non-differentiable models, Journal of Physics A: Mathematical and General, 38, 7, 1593-1613, 2005.

[36] Zacks, S and Even, M. The efficiencies in small samples of the maximum likelihood and best unbiased estimators of reliability functions, Journal of the American Statistical Association, 61, 316, 1033-1051, 1966. 